\title{
Savannah River Site Hazard Analysis Generic Initiator Database
}

by

C. R. Lux

Westinghouse Savannah River Company.

Savannah River Site

Alken, South Carolina 29808

D. S. Cramer
RECEIVED

JUN 111998

OSTI.

K. A. Wellmaker

S. R. Salaymeh

This paper was prepared in connection with work done under the above contract number with the U.S. Department of Energy. By acceptance of this paper, the publisher and/or recipient acknowledges the U.S. Government's right to retain a nonexclusive, royalty-free license in and to any copyright covering this paper, along with the right to reproduce and to authorize others to reproduce all or part of the copyrighted paper.
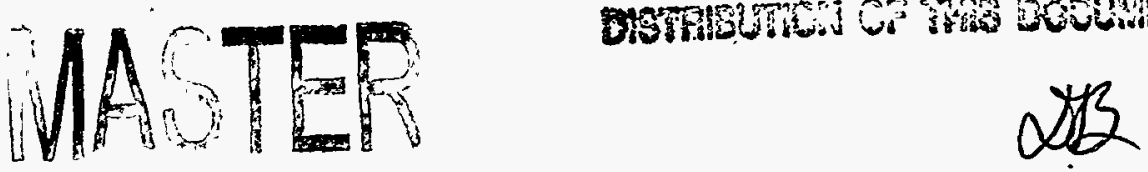


\section{1}

WSRC-RP-95-915

Rev. 0

\section{Savannah River Site}

\section{Hazard Analysis Generic Initiator Data Base (U)}

October 1995

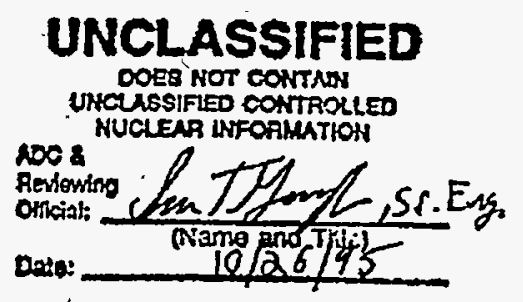

Westinghouse Savannah River Company Savannah River Site

Alken, SC 29808

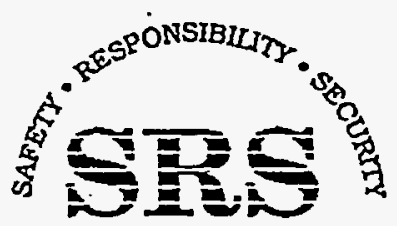

SAVANNAH RIVER SITE

PREPARED FOA THE U.S. DEPARTMENT OF ENERGY UNDER CONTRACT NO. DE-AC09-89SR18035 


\section{DISCLAIMER}

This report was prepared as an account of work sponsored by an agency of the United States Government. Neither the United States Government nor any agency thereof, nor any of their employees, makes any warranty, express or implied, or assumes any legal liability or responsibility for the accuracy, completeness, or usefulness of any information, apparatus, product, or process disclosed, or represents that its use would not infringe privately owned rights. Reference herein to any specific commercial product, process, or service by trade name, trademark, manufacturer, or otherwise does not necessarily constitute or imply its endorsement, recommendation, or favoring by the United States Government or any agency thereof. The views and opinions of authors expressed herein do not necessarily state or reflect those of the United States Government or any agency thereof.

This report has been reproduced directly from the best available copy.

Available to DOE and DOE contractors from the Office of Scientific and Technical Information, P. O. Box 62, Oak Ridge, TN 37831; prices available from (423) 576-8401.

Available to the public from the National Technical Information Service, U. S. Department of Commerce, 5285 Port Royal Road, Springfield, VA 22161. 


\section{DISCLAIMER}

Portions of this document may be illegible electronic image products. Images are produced from the best avaliable original document. 
WSRC-RP-95-915

Rev. 0

$\begin{array}{ll}\text { Key Words: } & \text { Frequency } \\ & \text { PHA } \\ & \text { Hozard Assessment } \\ & \text { HAD } \\ & \text { Bouncing } \\ & \\ \text { Retention } & \text { Permanent }\end{array}$

\section{Savannah River Site}

\section{Hazard Analysis Generic Initiator Data Base (U)}

by

C.R. Lux

D.S. Cramer

K.A. Wellmaker

S.R. Salaymeh

Issued: October 3, 1995 
WSRC-RP-95-915

Rev. 0

DOCUMENT: $\quad$ WSRC-RP-95-915, REV. 0

\section{TITLE: - SAVANNAH RIVER SITE HAZARDS ANALYSIS GENERIC INITIATOR DATA BASE (U)}

Approvals:

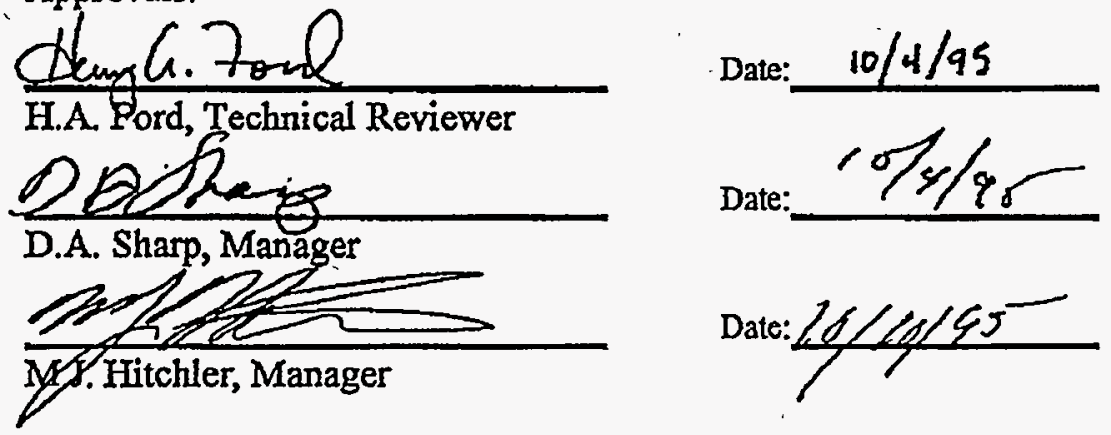


Rev. 0

CONTENTS

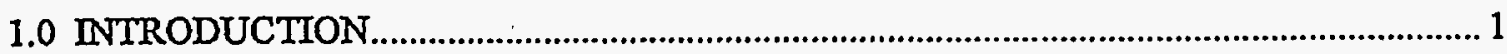

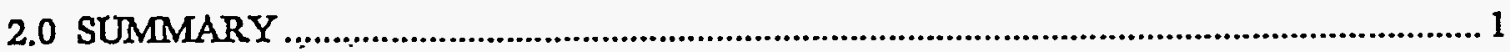

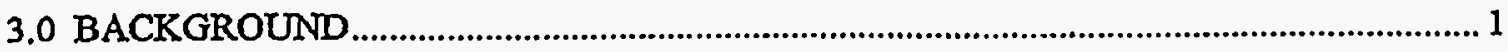

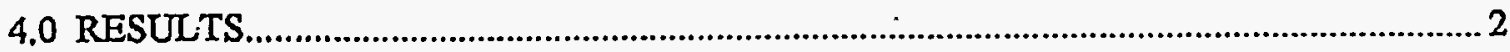

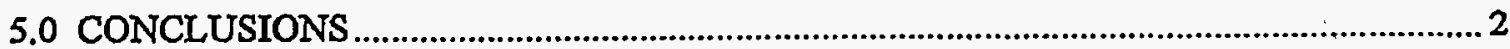

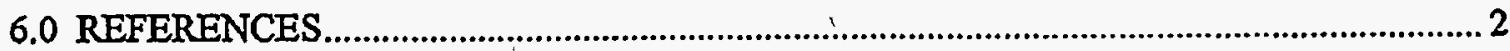

7.0 TABLES

8.0 TABLE 1 REFERENCES

TABLES

TABLE I HAZARD ANALYSIS GENERIC INITIATOR FREQUENCIES ............................ 3 


\subsection{INTRODUCTION}

Improved efficiency is a goal continually sought by most dedicated analysts. This has become more important as budgets have shrunk. An effort was made to examine the safety documentation process and determine areas where a change in the process could result in significant cost reductions. As a part of this Safety Documentation Integrated Work Process Task (Ref. 1), several cost saving initiatives were identified. This report addresses the cost saving initiative related to generic frequencies for hazards analyses.

\subsection{SUMMARY}

A set of "generic" frequencies for various common accidents within the Savannah River Site has been developed. These values provide a conservative or maximum frequency for each accident. This allows the use of the generic frequencies as a "go-no go" test. If the use of the generic frequency in the hazard analysis produces an acceptable result, no further frequency analysis is needed. This will reduce cost by minimizing the number of accidents which will require detailed frequency analysis.

This set of frequencies is being published as an official Westinghouse Savannah River Company (WSRC) report to provide a referenceable basis for assigning initiating frequencies in the hazards analyses.

\subsection{BACKGROUND}

The initial task involved identification of the types of accidents which lend themselves to generic frequencies. Care must be used when applying data to be certain that the conditions for which the data was collected is conservative for the application under consideration. For this reason, a " team was selected which consisted of two individuals familiar with the hazards analyses performed at the Savannah River Site (SRS) and two who were well versed in the interpretation and application of data. This mix allowed for selection of accidents typically addressed as a part of a hazards analysis and at the same time ensured that the frequencies would be of a conservative, maximum, and bounding nature.

In addition to accidents identified by the members of the team, a number of other sources were examined. The documents used included non-reactor Safety Analysis reports, the O \& M Methodology Manual, the Hazards Analysis Checklist, the reactor probabilistic safety analysis, and the Unreviewed Safety Question Checklist for Accidents.

The list was then examined for applicability to hazards analysis. Some of the items were stated too broadly to obtain a frequency for the event. In these cases, an attempt was made to determine what initiator(s) could be identified as associated with the broad category and provide its frequency(ies). Another problem encountered was a lack of data. Some potential initiators did not have sufficient data to allow determination of the initiator frequency. These initiators, if 
WSRC-RP-95-915

Rev. 0

the consequences are severe enough, will require more detailed analysis to determine their frequency.

\subsection{RESULTS}

Table 1 is a list of the identified generic hazard analysis frequencies. Each frequency has a reference and, as appropriate, comments associated with it (table references follow the table). The comments are intended to provide the user with some indication of the derivation of the frequency and, therefore, its applicability to the situation under consideration. For example, the "Red Oil" frequency is listed as incredible for F-Canyon given its controls. It would not be appropriate to assume all "Red Oil" reactions are incredible unless controls similar to those in FCanyon have been imposed. If more detail on the derivation of the frequency is desired, the reference may be consulted. It was intended that all frequencies be provided on a per hour basis. However, several initiators did not lend themselves well to the per hour unit. Sometimes a probability per demand, or accidents per mile was a far more useful unit. Therefore, the user is cautioned to examine each entry carefully to be certain that an appropriate application of the number is being made. Items for which data was not available at this time were labeled with "TBD" meaning To Be Determined. These items will be addressed in a future revision of this document.

\subsection{CONCLUSIONS}

The benefits from this effort will come in three areas. The frequencies assigned during the hazards analysis process will be more defensible, some hazards analysis effort will be saved, and some accident analysis effort will be eliminated due to the accident being binned out at the hazards analysis level. It is estimated that approximately 5 hazards analyses will be performed per year. The hazards analysis time will be reduced by about 24 man hours per analysis or 3 man-weeks per year. The screening process will now eliminate about 1 more accident than it would have previously. The entire accident analysis cycle requires 4 man-weeks. Thus, there will be an accident analysis savings of 20 man-weeks. The total savings will be 23 man-weeks or about 0.5 Full Time Equivalents.

\subsection{REFERENCES}

1 Lamb, V.F., et. al. Safety Documentation Integrated Work Process Task, Final Report (U). WSRC-TR-95-0279, Revision 1, Westinghouse Savannah River Company, Aiken, SC, July 1995. 
WSRC-RP-95-915

Rey. 0

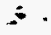

7.0 TABLES

-3 - 
Table 1 Hazard Analysis Generic Initiator Frequencies

\begin{tabular}{|c|c|c|c|c|c|}
\hline 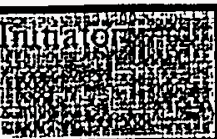 & 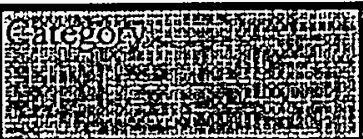 & 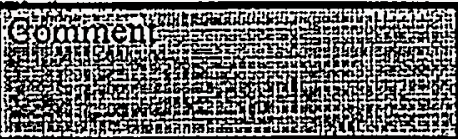 & 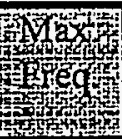 & 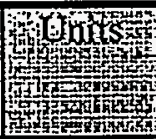 & 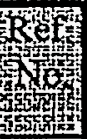 \\
\hline Air Pollution & Air Stagnation & Site Meteorology & 2 & /year & 1 \\
\hline Airplane & Crash & $\begin{array}{l}\text { For Onsite Area of } 120 \text { acres } \\
\left(0.19 \mathrm{mi}^{2}\right)\end{array}$ & $<2 \mathrm{E}-7$ & /year & 2 \\
\hline Bearings & All & bearings, Ball, Heavy Duty & $2 \mathrm{E}-5$ & /hour & 3 \\
\hline Belts & All & Belts, Drive & $4 \mathrm{E}-5$ & /hour & 3 \\
\hline Truck & $\begin{array}{l}\text { Haz. Material } \\
\text { Movement Accident }\end{array}$ & $\begin{array}{l}\text { From Site Transportation } \\
\text { SAR }\end{array}$ & $6 \mathrm{E}-06$ & $\begin{array}{l}\text { accidents } \\
\text { /mile }\end{array}$ & 4 \\
\hline Cell Cover & Collapse & See Drop, Human Error & - & - & - \\
\hline $\begin{array}{l}\text { Charge/Disch } \\
\text { arge }\end{array}$ & Errors, Misloading & TBD & - & - & - \\
\hline \multirow[t]{2}{*}{ Chemical } & $\begin{array}{l}\text { Uncontrolled } \\
\text { Chemical Reactions }\end{array}$ & F-Canyon SAR. Total & 2 & lyear & 5 \\
\hline & Red Oil & $\begin{array}{l}\text { Based on F Canyon } \\
\text { Operations with SCl's }\end{array}$ & $<1 \mathrm{E}-6$ & /year & $\begin{array}{c}6,7, \\
8\end{array}$ \\
\hline Chlorine & All & See Gases & - & - & - \\
\hline \multirow{2}{*}{$\begin{array}{l}\text { Coil and } \\
\text { Tube }\end{array}$} & Leak & F-Canyon & 1 & /year & 5 \\
\hline & Rupture & PRA for USF & $8 \mathrm{E}-3$ & /yr/coil & 9 \\
\hline Cold & Ice, Snow, Wind, etc. & See Specific Category & - & - & - \\
\hline Collapse & \begin{tabular}{|l} 
Building or Wall \\
(Non-Seismic)
\end{tabular} & TBD & - & - & - \\
\hline Combustible & Combustible Material & See Fire & - & - & - \\
\hline
\end{tabular}


Table 1 Hazard Analysis Generic Initiator Frequencies (Continued)

\begin{tabular}{|c|c|c|c|c|c|}
\hline 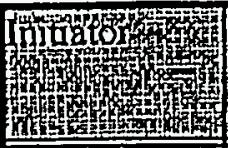 & 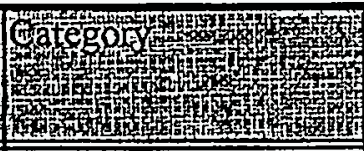 & 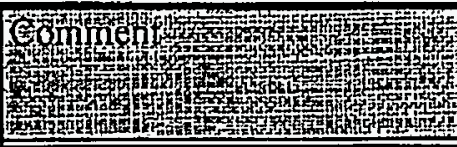 & 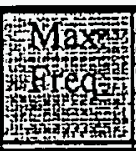 & H. & 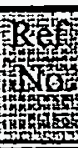 \\
\hline $\begin{array}{l}\text { Common } \\
\text { Cause }\end{array}$ & General & $\begin{array}{l}\text { Use Beta Factor of } 0.1 . \mathrm{F} \\
\text { stands for general frequency } \\
\text { of initiator. }\end{array}$ & $F \times 0.1$ & Freq. & 10 \\
\hline \multirow[t]{16}{*}{ Confinement } & Coil and Tube & See Coil, Heat Exchangers & - & - & - \\
\hline & Filters & See Filter & - & - & - \\
\hline & Fuel Leakage & See Pipe, Tank, etc. & $\overline{-}$ & - & - \\
\hline & Gasket/Seal & See Leak & - & - & - \\
\hline & Pipe & See Pipe & - & - & - \\
\hline & Shipping Container & See Shipping Container & - & $\overline{-}$ & - \\
\hline & Valve & See Valve & - & - & - \\
\hline & Vessel & See Vessel & - & - & - \\
\hline & Pipe Break & See Pipe & - & - & - \\
\hline & Piping Error & See Human Error & 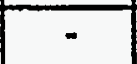 & - & - \\
\hline & Pluggage & See Pluggage & - & - & - \\
\hline & $\begin{array}{l}\text { Premature or } \\
\text { Excessive Transfers }\end{array}$ & See Human Error & - & - & - \\
\hline & Siphoning & See Siphoning & $\overline{-}$ & - & - \\
\hline & Suckback & See Suckback & - & - & - \\
\hline & Valving Error & See Human Error & - & - & - \\
\hline & Vessel & See Vessel & - & - & - \\
\hline $\begin{array}{l}\text { Control } \\
\text { circuit }\end{array}$ & AlI & See Electrical & - & - & - \\
\hline Corrosion & General & TBD & - & - & - \\
\hline
\end{tabular}


Table 1 Hazard Analysis Generic Initiator Frequencies (Continued)

\begin{tabular}{|c|c|c|c|c|c|}
\hline 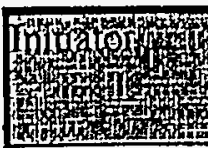 & 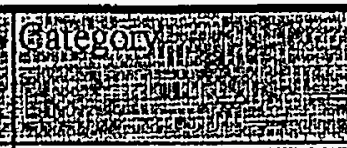 & 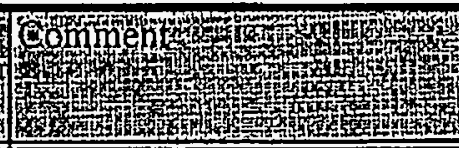 & 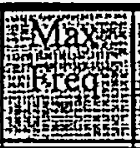 & 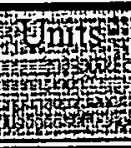 & 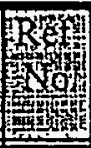 \\
\hline Crane & Drop & See Drop & - & - & - \\
\hline Criticality & All & $\begin{array}{l}\text { Situationally dependent, but } \\
\text { with double contingencies } \\
\text { present. }\end{array}$ & $<1 \mathrm{E}-2$ & /year & Esti \\
\hline$\overline{\text { Dams }}$ & All & UCRL-15910 & $\overline{1 \mathrm{E}-4}$ & Tyear & 11 \\
\hline Debris & All & See Human Error & - & - & - \\
\hline Diesel & Non-Electrical & Site Generic. Fan & $5 \mathrm{E}-3$ & $\mathrm{hr}$ & 12 \\
\hline $\begin{array}{l}\text { Distributed } \\
\text { Control } \\
\text { System }\end{array}$ & All & See Electrical & - & - & - \\
\hline \multirow[t]{3}{*}{ Drop } & $\begin{array}{l}\text { Cranes, Hoists, } \\
\text { forklifts, etc. }\end{array}$ & TBD & - & - & - \\
\hline & Fuel Bundle & TBD & - & - & - \\
\hline & Human Error & See Human Error & $\overline{-}$ & $\overline{-}$ & - \\
\hline \multirow[t]{5}{*}{ Earthquake } & $\overline{P C O}$ & Not Applicable & - & - & 13 \\
\hline & $\overline{\mathrm{PC} 1,0.1 \mathrm{~g}}$ & & $\overline{2 \mathrm{E}-3}$ & Tyear & 13 \\
\hline & $\overline{\mathrm{PC} 2,0.13 \mathrm{~g}}$ & & 1E-3 & /year & 13 \\
\hline & PC3, 0.18g & & $5 \mathrm{E}-4$ & /year & 13 \\
\hline & PC4, 0.32g & & IE-3 & /year & 13 \\
\hline \multirow[t]{3}{*}{ Electrical } & $\begin{array}{l}\text { Automatic Transfer } \\
\text { Switches }\end{array}$ & Spurious Transfer & $3 \mathrm{E}-2$ & lyear & 14 \\
\hline & Battery, Liquid & Reactor PRA work & 0.1 & /year & 15 \\
\hline & Battery, Solid & Reactor PRA work & 0.1 & /year & 15 \\
\hline
\end{tabular}


Table 1 Hazard Analysis Generic Initiator Frequencies (Continued)

\begin{tabular}{|c|c|c|c|c|c|}
\hline 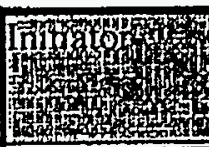 & 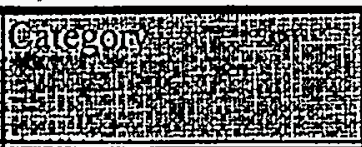 & 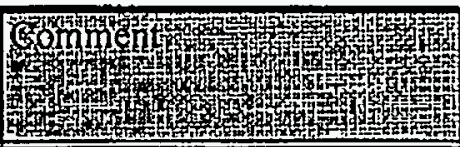 & 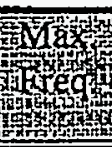 & 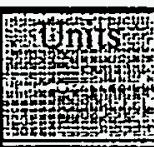 & Ref \\
\hline \multirow[t]{17}{*}{$\begin{array}{l}\text { Electrical } \\
\text { (Continued) }\end{array}$} & $\begin{array}{l}\text { Breaker:Spurious } \\
\text { Operation }\end{array}$ & Reactor PRA work & $\overline{5 E-3}$ & /year & 15 \\
\hline & Bus. & Reactor PRA-work & $2 \mathrm{E}-2$ & /year & 15 \\
\hline & Cable Runs & Cables, 1 to $33 \mathrm{kv}$ & $4 \mathrm{E}-6$ & /hour/km & 3 \\
\hline & Control Circuit & $\begin{array}{l}\text { See Distributed Control } \\
\text { System }\end{array}$ & $\because$ & - & - \\
\hline & Diesel with Generator & K-Reactor PRA & 20 & /year & 10 \\
\hline & $\begin{array}{l}\text { Distributed Control } \\
\text { System }\end{array}$ & OREDA & 1 & /year & 16 \\
\hline & Fuse & Site Generic & 1E-7 & thour & 12 \\
\hline & Featers & & $1 \mathrm{E}-5$ & thour & 12 \\
\hline & Hot Plates & See Heater & - & - & - \\
\hline & Interlock & Page 608 & $3 \mathrm{E}-7$ & thour & 17 \\
\hline & Lightning:Damaging & $\begin{array}{l}\text { Damages Safety Equipment. } \\
\text { Reactor PRA work. }\end{array}$ & 0.2 & $\begin{array}{c}\text { |yr/ } \\
\text { facility }\end{array}$ & 15 \\
\hline & Lightning:General & $\begin{array}{l}\text { Site Climatology. } 4 \text { in } \\
\text { summer }\end{array}$ & $\overline{6}$ & fyr & 1 \\
\hline & $\begin{array}{l}\text { Locomotive Engine } \\
\text { Wreck }\end{array}$ & See Train & - & - & - \\
\hline & Motor & See Motor & - & - & - \\
\hline & Power & See Power Loss & - & - & - \\
\hline & Power Supply & Page 333 & $1 \mathrm{E}-6$ & /hour & 17 \\
\hline & Pumps & See Púmp & $=$ & - & - \\
\hline
\end{tabular}


Table 1 Hazard Analysis Generic Initiator Frequencies (Continued)

\begin{tabular}{|c|c|c|c|c|c|}
\hline 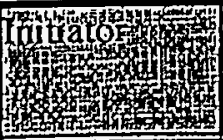 & 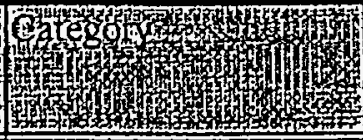 & 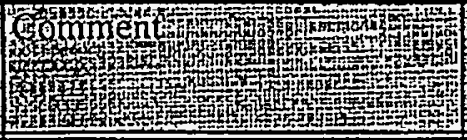 & 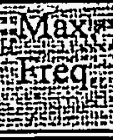 & 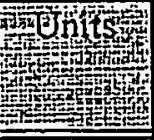 & Nefe \\
\hline \multirow{13}{*}{$\begin{array}{l}\text { Electrical } \\
\text { (Continued) }\end{array}$} & Relays & Reactor PRA work. & $5 \mathrm{E}-2$ & /year & 15 \\
\hline & Service Outlets & TBD & - & - & - \\
\hline & $\begin{array}{l}\text { Short Phase to Phase } \\
\text { (Hot Shorts) }\end{array}$ & $\begin{array}{l}\text { One Foot Long Pair. Reactor } \\
\text { PRA work. }\end{array}$ & $5 \mathrm{E}-8$ & /yr/pair & 18 \\
\hline & Short to Ground & Reactor PRA work. & 1E-7 & /yr/foot & 18 \\
\hline & Static Electricity & TBD & - & - & - \\
\hline & Switchgear & $\begin{array}{l}\text { See Automatic Transfer } \\
\text { Switch }\end{array}$ & - & - & - \\
\hline & Thermocouples & Reactor PRA work. & $5 \mathrm{E}-2$ & lyear & 15 \\
\hline & $\begin{array}{l}\text { Transformers: All } \\
\text { Failure Mode }\end{array}$ & Site Generic & $1 \mathrm{E}-6$ & Mour & 12 \\
\hline & Transformers: Fires & & $8 \mathrm{E}-4$ & /year & 19 \\
\hline & Transmission Lines & Page 741 & $2 \mathrm{E}-7$ & /cycle/ft & 17 \\
\hline & Underground Wiring & Page 771 & $7 \mathrm{E}-9$ & /cycle/ft & 17 \\
\hline & Welding (Electrical) & See Fire & - & - & - \\
\hline & Wiring & Page 757 & $4 \mathrm{E}-9$ & /cycle/ft & 17 \\
\hline \multirow{2}{*}{$\begin{array}{l}\text { Expansion } \\
\text { Joint }\end{array}$} & Leak & K-Reactor PRA. & $3 \mathrm{E}-2$ & lyear & 10 \\
\hline & Catastrophic & $\begin{array}{l}\text { Sec. 2.1.3, 95 Methodology } \\
\text { Manual }\end{array}$ & $3 E-4$ & lyear & 20 \\
\hline \multirow[t]{3}{*}{ Explosion } & Chemical Reactions & See Chemical & - & - & - \\
\hline & Pressure Release & See Pipe or Vessel & - & - & - \\
\hline & Gases/Vapors & Based on Tritium Data & 2 & lyear & 21 \\
\hline
\end{tabular}


Table 1 Hazard Analysis Generic Initiator Frequencies (Continued)

\begin{tabular}{|c|c|c|c|c|c|}
\hline 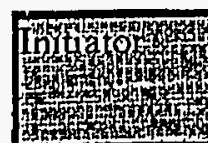 & \begin{tabular}{|c|c|} 
& \\
\end{tabular} & 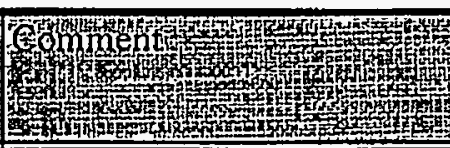 & The & 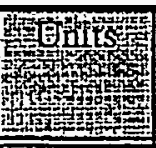 & \\
\hline Explosion & Metal:Powder & $\overline{\mathrm{TBD}}$ & - & - & $\overline{-}$ \\
\hline & Metal:Solid & TBD & - & - & - \\
\hline & $\begin{array}{l}\text { Red Oil or Nitration } \\
\text { reactions }\end{array}$ & See Chemical:Red Oil & - & - & - \\
\hline & Resin Column & See Uncontrolled Reactions & - & - & - \\
\hline $\begin{array}{l}\text { Fabrication } \\
\text { Error }\end{array}$ & All & See Human Error & - & - & - \\
\hline$\overline{\text { Falling }}$ & $\begin{array}{l}\text { Collapse (building or } \\
\text { wall collapse) }\end{array}$ & See Collapse or Earthquake & - & - & - \\
\hline Fans & Spark & $\begin{array}{l}\text { Sec. 2.1.6, 95 Methodology } \\
\text { Manual }\end{array}$ & $3 \mathrm{E}-2$ & lyear & 20 \\
\hline & Fail to Run & Site Generic. Motor Driven & $3 \mathrm{E}-5$ & thour & 12 \\
\hline Filter & $\begin{array}{l}\text { Emergency Water } \\
\text { Cooling:Plug }\end{array}$ & Site Genieric. & $3 \mathrm{E}-6$ & hour & 12 \\
\hline & $\begin{array}{l}\text { Emergency Water } \\
\text { Cooling:Catastrophic }\end{array}$ & Site Generic. & $5 \mathrm{E}-7$ & /hour & 12 \\
\hline & HEPA & ITP Purge System & $1 \mathrm{E}-2$ & /year & 22 \\
\hline & Liquid & TBD & - & - & - \\
\hline & Sand & $\begin{array}{l}\text { Penetration Factor. Always } \\
\text { Available }\end{array}$ & $5 \mathrm{E}-3$ & Fraction & 23 . \\
\hline Fire & Process Related & F-Canyon SAR & $1 \mathrm{E}-6$ & thour & 5 \\
\hline & Forest & TBD & - & - & - \\
\hline & Motor & See Motor & - & - & - \\
\hline
\end{tabular}


Table 1 Hazard Analysis Generic Initiator Frequencies (Continued)

\begin{tabular}{|c|c|c|c|c|c|}
\hline 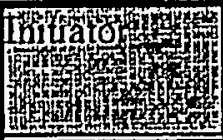 & 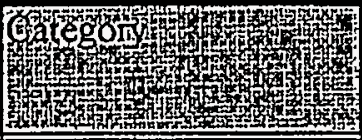 & 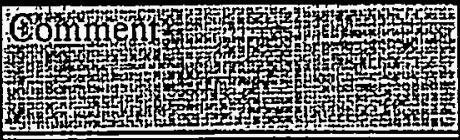 & 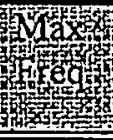 & 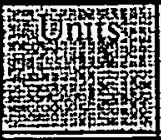 & 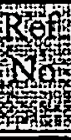 \\
\hline $\begin{array}{l}\text { Fissile } \\
\text { Material }\end{array}$ & All & See Criticality & - & - & - \\
\hline Flammables & All & See Fire & - & - & - \\
\hline Flood & All & See Rain or Dam & - & - & - \\
\hline \multirow[t]{2}{*}{ Forklift } & Drop & See Drop & - & - & - \\
\hline & Wreck & See Human Error & - & - & - \\
\hline Freezing & All & See Ice & - & - & - \\
\hline Friction & All & See Valve, Pump, Fire,... & - & - & - \\
\hline Fuel & Bad Diesel/Gasoline & K-Reactor PRA. & $4 \mathrm{E}-2$ & lyear & 10 \\
\hline \multirow[t]{4}{*}{ Gases } & Fire & See Fire & - & - & - \\
\hline & Release:Tritium & Based on Tritium Facility & 4 & events/ yr & 21 \\
\hline & Release:Chlorine & Site Data & $2 \mathrm{E}-4$ & $\mid \begin{array}{c}\text { year/are } \\
\text { a }\end{array}$ & 24 \\
\hline & Explosion & See Explosion & - & - & - \\
\hline Gear & General & Gear, Helical & $1 E-6$ & /hour & 3 \\
\hline Hail/Sleet & All & Site Meteorology & 0.5 & /year & 1 \\
\hline Halon & All & See Gases:Release & - & - & - \\
\hline \multirow[t]{3}{*}{$\begin{array}{l}\text { Heat } \\
\text { Exchanger }\end{array}$} & $\begin{array}{l}\text { Leak:Internal (several } \\
\text { gpm) }\end{array}$ & 95 Methodology Manual & $2 \mathrm{E}-2$ & /year & 20 \\
\hline & Leak:External & See Pipe, Vessel & - & - & - \\
\hline & All Other (Plug,etc.) & See Filter, EWC:Plug & - & - & - \\
\hline Helicopter & Crash:Offsite & See Airplane & - & - & - \\
\hline
\end{tabular}


Rev. 0

Table 1 Hazard Analysis Generic Initiator Frequencies (Continued)

\begin{tabular}{|c|c|c|c|c|c|}
\hline 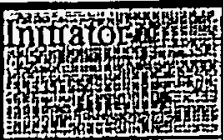 & 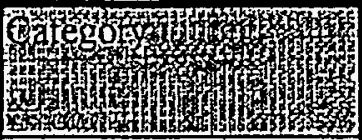 & 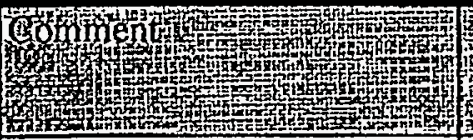 & 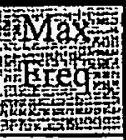 & 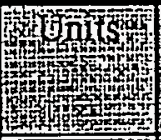 & ref \\
\hline \multirow{2}{*}{$\begin{array}{l}\text { Helicopter } \\
\text { Crash }\end{array}$} & Crash:Onsite & General & .5 & /year & 25 \\
\hline & $\begin{array}{l}\text { Crash:Onsite into } \\
\text { Building }\end{array}$ & $\begin{array}{l}\text { Based on DWPF Location } \\
\text { Relative to B Area }\end{array}$ & $1 \mathrm{E}-10$ & \begin{tabular}{|c|}
$\mathrm{ft}^{2}$ of \\
Effective \\
Target \\
Area
\end{tabular} & 26 \\
\hline Hoists & Drop & See Drop & - & - & - \\
\hline Hot Shorts & Electrical & See Electrical:Phase to Phase & - & - & - \\
\hline \multirow[t]{4}{*}{ Human Error } & High & $\begin{array}{l}\text { Based on Supervisor } \\
\text { Yerification }\end{array}$ & .5 & /Demand & 27 \\
\hline & Moderate & $\begin{array}{l}\text { Based on Supervisor } \\
\text { Verification }\end{array}$ & .3 & /Demand & 27 \\
\hline & Low & $\begin{array}{l}\text { Based on Supervisor } \\
\text { Verification }\end{array}$ & .1 & /Demand & 27 \\
\hline & Overflow & & $5 \mathrm{E}-5$ & $/ \operatorname{tank}-\mathrm{hr}$ & 27 \\
\hline Hurricane & All & $\begin{array}{l}\text { Site Meteorology. Within } \\
\text { State. (Once every } 8 \text { years) }\end{array}$ & 0.1 & /year & 1 \\
\hline \multirow[t]{2}{*}{ Ice } & $\begin{array}{l}\text { Accumulation on } \\
\text { Surfaces }\end{array}$ & Site Climatology & 0.5 & /year & 1 \\
\hline & 0.5 in accumulation & Site Climatology & $4 \mathrm{E}-2$ & /year & 1 \\
\hline Ignition & Gases/Vapor, Liquids & See Fire & - & - & - \\
\hline Interlock & All & See Electrical & - & - & - \\
\hline Leak & All & See Pipe, Vessel,... & - & - & - \\
\hline Lifts & Drop & See Drop & - & - & - \\
\hline Lightning & All & See Electrical & - & - . & - \\
\hline
\end{tabular}


Table 1 Hazard Analysis Generic Initiator Frequencies (Continued)

\begin{tabular}{|c|c|c|c|c|c|}
\hline 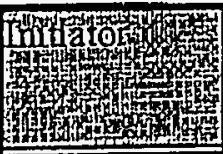 & 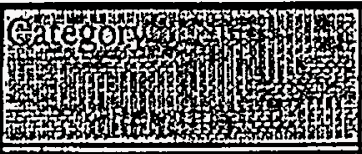 & 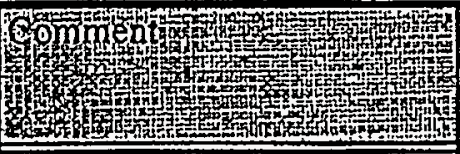 & 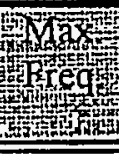 & 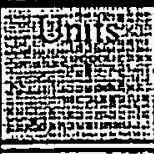 & the \\
\hline Line Breaks & Pipe & See Pipe & - & - & - \\
\hline Meteorite & All & & 1E-9 & lyear & 28 \\
\hline \multirow[t]{2}{*}{ Missile } & Compressed Gas & TBD & - & - & - \\
\hline & Turbine & TBD & - & - & - \\
\hline $\begin{array}{l}\text { Missile } \\
\text { (continued) }\end{array}$ & Tormado & See Tornado & - & - & - \\
\hline Motion & $\begin{array}{l}\text { Vehicles/forklifts and } \\
\text { trucks }\end{array}$ & See Vehicle, Drop, etc. & - & - & - \\
\hline \multirow[t]{2}{*}{ Motors } & Fire & TBD & - & - & - \\
\hline & Fails to Run & Site Generic. DC. & $1 \mathrm{E}-5$ & /hour & 12 \\
\hline $\begin{array}{l}\text { Natural } \\
\text { Phenomena }\end{array}$ & Natural Phenomena & See Earthquake,Tornado,... & - & - & - \\
\hline $\begin{array}{l}\text { Nuclear } \\
\text { Criticality }\end{array}$ & All & See Criticality & - & - & - \\
\hline Overflow & All . & See Human Error & - & - & - \\
\hline $\begin{array}{l}\text { Overpressuri } \\
\text { zation }\end{array}$ & Human & See Human Error & - & - & - \\
\hline \multirow[t]{4}{*}{ Pipe } & Catastrophic & 95 Methodology Manual & $1 E-6$ & /ft/year & 20 \\
\hline & Leak Size & 95 Methodology Manual & $2 \mathrm{E}-5$ & /ft/year & 20 \\
\hline & Pipe Size & 95 Methodology Manual & $2 \mathrm{E}-5$ & /ftyear & 20 \\
\hline & Pluggage & Reactor PRA work. & $1 \mathrm{E}-6$ & lyear & 29 \\
\hline \multirow[t]{2}{*}{ Power Loss } & $\begin{array}{l}\text { Normal \& } \\
\text { Emergency/480V }\end{array}$ & TBD & - & - & - \\
\hline & Normal/480V & TBD & - & - & - \\
\hline
\end{tabular}


Table 1 Hazard Analysis Generic Initiator Frequencies (Continued)

\begin{tabular}{|c|c|c|c|c|c|}
\hline 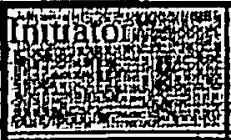 & 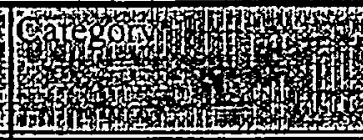 & 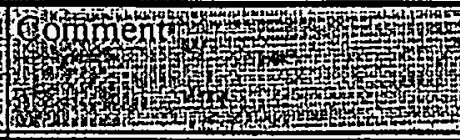 & 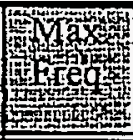 & 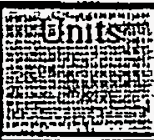 & re \\
\hline \multirow{2}{*}{$\begin{array}{l}\text { Power Loss } \\
\text { (continued) }\end{array}$} & Normal/12.8KV & TBD & - & - & - \\
\hline & Normal/Area 115KV & TBD & - & - & - \\
\hline \multirow[t]{4}{*}{ Pressure } & Gas Bottles & See Missile, Gases:Release & - & - & - \\
\hline & $\begin{array}{l}\text { Gas } \\
\text { Receivers/Reservoir }\end{array}$ & See Overpressurization & - & - & - \\
\hline & Pressure vessels & See Pipes, Vessel & - & - & - \\
\hline & $\begin{array}{l}\text { Steam headers and } \\
\text { Lines }\end{array}$ & See Steam Tube & - & - & - \\
\hline Pump & Fails to Run & $\begin{array}{l}\text { Site Generic. Motor or } \\
\text { Turbine Driven. }\end{array}$ & $1 \mathrm{E}-4$ & /hour & 12 \\
\hline $\begin{array}{l}\text { Pyrophoric } \\
\text { Material }\end{array}$ & All & See Fire & - & - & - \\
\hline $\begin{array}{l}\text { Radiation, } \\
\text { lonizing }\end{array}$ & All & See Criticality & - & - & - \\
\hline Radiolysis & All & See Explosion or Fire & - & - & - \\
\hline \multirow[t]{2}{*}{ Rain } & High $(>\sin / 24 \mathrm{hrs})$ & Site Climatology & 0.2 & /year & 1 \\
\hline & $\begin{array}{l}\text { Moderate(>3 in } / 24 \\
\text { hrs). }\end{array}$ & Site Climatology & 1 & lyr & \\
\hline Red Oil & All & See Chemical & - & - & - \\
\hline Rigging & Drop & See Drop & - & - & - \\
\hline $\begin{array}{l}\text { Roof or Wall } \\
\text { Collapse }\end{array}$ & All & See Earthquake or Collapse & - & - & - \\
\hline Rupture & All & See Pipe, Vessel,... & - & - & - \\
\hline
\end{tabular}


Table 1 Hazard Analysis Generic Initiator Frequencies (Continued)

\begin{tabular}{|c|c|c|c|c|c|}
\hline 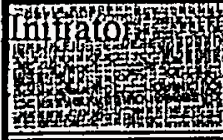 & 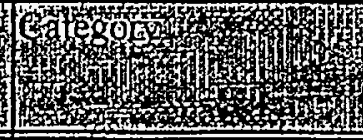 & 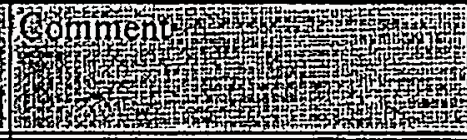 & 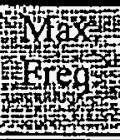 & H. & me \\
\hline Rust & All & See Corrosion & - & - & - \\
\hline $\begin{array}{l}\text { Shorts to } \\
\text { Ground }\end{array}$ & All & See Electrical & - & - & - \\
\hline Siphoning & Liquid & F-Canyon SAR. & $2 \mathrm{E}-5$ & /hour & 5 \\
\hline Sleet/Hail & All & See Hail/Sleet & - & - & - \\
\hline \multirow[t]{3}{*}{ Snow } & $>12$ inches & Max 13 inches in 1973 & $5 E-2$ & lyear & 1 \\
\hline & 1 inch or more & Site Climatology & 0.2 & /year & 1 \\
\hline & $\begin{array}{l}\text { Time Snow Remains } \\
\text { on Ground }\end{array}$ & $\begin{array}{l}\text { Site Climatology, Rarely } \\
\text { Exceeds }\end{array}$ & 3 & days & 1 \\
\hline Spill & Liquid & $\begin{array}{l}\text { F-Canyon SAR Leak., } \\
\text { Overflow }\end{array}$ & $7 \mathrm{E}-3$ & /hour & 5 \\
\hline $\begin{array}{l}\text { Spontaneous } \\
\text { Combustion }\end{array}$ & All & See Fire & - & - & - \\
\hline Stack failure & All & See Collapse & - & - & - \\
\hline \multirow[t]{2}{*}{ Steam Tube } & Leak & See Coil \& Tube Leak & - & - & - \\
\hline & Catastrophic Failure & See Coil \& Tube Rupture & - & - & - \\
\hline Straight wind & All & See Tornado & - & - & - \\
\hline Structural & Collapse & See Collapse & - & - & - \\
\hline Suckback & All & See Siphoning & - & - & - \\
\hline Tank & All & See Vessel & - & - & - \\
\hline \multirow[t]{2}{*}{ Temperature } & Ambient:High & Site Climatology. $>100^{\circ} \mathrm{F}$ & 1 & lyear & 1 \\
\hline & Ambient:Low & See Ice & - & - & - \\
\hline Thermal & All & See Fire & - & - & - \\
\hline
\end{tabular}


WSRC-RP-95-915

Rev. 0

Table 1 Hazard Analysis Generic Initiator Frequencies (Continued)

\begin{tabular}{|c|c|c|c|c|c|}
\hline 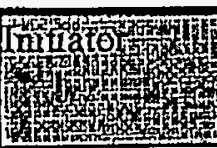 & 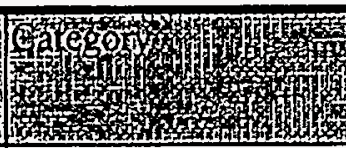 & 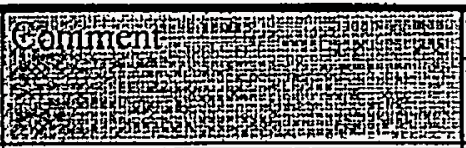 & 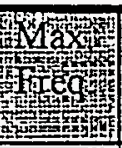 & 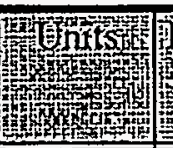 & the \\
\hline $\begin{array}{l}\text { Thunder- } \\
\text { storms }\end{array}$ & Average & $\begin{array}{l}\text { Site Climatology. Most June, } \\
\text { July \& August }\end{array}$ & 5 & /month & 1 \\
\hline \multirow[t]{6}{*}{ Tomado } & $\mathrm{PCO}$ & Not Applicable & - & - & $\overline{13}$ \\
\hline & $\overline{\mathrm{PCl}}$ & Not Applicable & - & - & 13 \\
\hline & $\overline{\mathrm{PC} 2}$ & Not Applicable & - & - & 13 \\
\hline & PC3 & $137 \mathrm{mph}, 40 \mathrm{psf} @ 20 \mathrm{psf} / \mathrm{sec}$ & $2 \mathrm{E}-5$ & /year & 13 \\
\hline & $\mathrm{PC4}$ & $\begin{array}{l}\text { 192mph, } 125 \text { psf@50 } \\
\text { psf/sec }\end{array}$ & $2 \mathrm{E}-6$ & lyear & 13 \\
\hline & $>155 \mathrm{mph}$ & & $2 \mathrm{E}-5$ & /year & 30 \\
\hline \multirow[t]{2}{*}{ Train } & Derail & Exceeding $60 \mathrm{ft}$. from track & $<1 \mathrm{E}-6$ & /yr & $31^{1}$ \\
\hline & $\begin{array}{l}\text { Haz. Material } \\
\text { Movement Accident }\end{array}$ & $\begin{array}{l}\text { From Site Transportation } \\
\text { SAR }\end{array}$ & $1 \mathrm{E}-5$ & \begin{tabular}{|l|} 
accidents \\
mile
\end{tabular} & 4 \\
\hline $\begin{array}{l}\text { Transfer } \\
\text { Error }\end{array}$ & All & See Human Error & - & - & - \\
\hline $\begin{array}{l}\text { Trench } \\
\text { Cover }\end{array}$ & All & See Drop, Human Error & - & - & - \\
\hline $\begin{array}{l}\text { Tripping, } \\
\text { Slipping }\end{array}$ & All & See Human Error & - & - & - \\
\hline $\begin{array}{l}\text { Tube and } \\
\text { Coil }\end{array}$ & Leak & See Coil \& Tube Leak & - & - & - \\
\hline Turbines & Missiles & See Missile & - & - & - \\
\hline $\begin{array}{l}\text { Uncontrolled } \\
\text { Chemical } \\
\text { Reactions }\end{array}$ & All & See Chemical & - & - & - \\
\hline
\end{tabular}


Rev. 0

Table 1 Hazard Analysis Generic Initiator Frequencies (Continued)

\begin{tabular}{|c|c|c|c|c|c|}
\hline 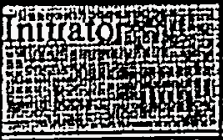 & 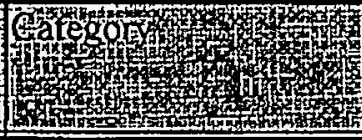 & 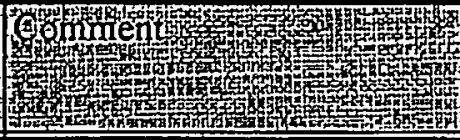 & 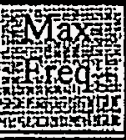 & The & 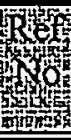 \\
\hline \multirow[t]{4}{*}{ Vehicle } & Airplane & See Airplane & - & - & - \\
\hline & Helicopter & See Helicopter & - & - & - \\
\hline & Train & See Train & - & - & - \\
\hline & Truck/Car & See Car/Truck & - & - & - \\
\hline \multirow[t]{2}{*}{ Vessel } & Leak & Site Generic. Unpressurized & 1E-7 & Thour & 12 \\
\hline & Catastrophic & Site Generic. Unpressurized & $5 \mathrm{E}-9$ & /hour & 12 \\
\hline Vibration & Mechanical & See Motor, Pipe, Valve,... & - & - & - \\
\hline Volcanoes & All & - & $<1 E-6$ & lyear & 32 \\
\hline Water & All & $\begin{array}{l}\text { See Dam, Rain, Leaks, } \\
\text { Spills,... }\end{array}$ & - & - & - \\
\hline Weather & All & See Type of Weather & - & - & - \\
\hline \multirow[t]{3}{*}{ Wind } & High, Straight & See Tornado & - & - & - \\
\hline & Hurricane & See Hurricane & - & - & - \\
\hline & Tornado & See Tomado & - & - & - \\
\hline
\end{tabular}


WSRC-RP-95-915

Rev. 0

\subsection{TABLE 1 REFERENCES}

1. Hunter, C.H. A Climatological Descrìntion of the Savannah River Site. WSRC-RP-89313, Westinghouse Savannah River, Aiken, SC, May 1990.

2 Lux, C.R. Frequency of a Aircraft Crash in the Burial Ground (U). Calc-Note S-CLC-E0067. Westinghouse Savannah River, Aiken, SC, July 1995.

3 Dexter, A.H., and Perkins, W.C. Component Failure-Rate Data with Potential Applicability to a Nuclear Fuel Reprocessing Plant. E.I. du Pont de Nemours and Company, Savannah River Laboratory, July 1982.

4 Evaluations of Accident Risks in the Transportation of Hazards Materials by Truck and Rail at the Savannah River Site (U). WSRC-RP-89-715, Revision 1, Westinghouse Savannah River Company, Aiken, SC. September 1992.

5 E-Canyon Safety Analysis Report(U). DPSTSA-200-10 SUP-4, 1993, and Addendum \#2 and \#4, Rev.1, Westinghouse Savannah River Company, Aiken, SC, July 1994.

6 Lux, C.R., Christiansen, L.W., Marshall, K.M., and Slaven, T. Frequency Determination for Runaway TBPANitric Acid Reactions in Support of the F-Canyon BIO (U). S-CLC-F00100, Revision 1, Westinghouse Savannah River Company, Aiken, SC, June 1994.

7 Christiansen, L.W., and Lux, C.R. Fault Tree Analysis of Red Oil Reactions in the FCanyon Evaporators (U). S-CLC-F-00140, Revision B, Westinghouse Savannah River Company, Aiken, SC, March 1995.

8 Vail, R.E. to Lux, C.R., et. al. Human Factors Review of the "Red Oil" Explosion Fault Trees for the F Canyon Evaporators. EPD-SIM-940098, Westinghouse Savannah River, Aiken, SC, October 1994.

9 Cramer, D.S. Database for Probabilistic Risk Assessment of the Uranium Solidification Facility (U). SRT-DCA-93002, Westinghouse Savannah River Company, Aiken, SC, November 1993.

10 Brandyberry, M.D., Cramer, D.S., et. al. SRS PRA of Reactor Operation - Level 1. Internal Events. Westinghouse Sivannah River Company, Aiken, SC, June 1990.

11 Kennedy, R.P., et. al. Design and Evaluation Guidelines for Department of Energy Facilities Subjected to Natural Phenomena Hazards. UCRL-15910, United States Department of Energy, Washington, D.C., June 1990.

12 Blanton, C.H. and Eide, S.A. (LATA). Savannah River Site Generic Data Base Development (U). WSRC-TR-93-262, Westinghouse Savannah River Company, Aiken, SC, June 1993.

13 Natural Phenomena Hazards Design and Evaluation Criteria for Department of Energy Facilities. DOE-STD-1020-94, U.S. Department of Energy, Washington, D.C., April 1994. 
14 Cramer, D.S. to PRA File, Reactor Risk Analysis Group. Failure, Modes and Rates for Automatic Transfer Switches. SRL-PRA-910276, E.I. du Pont de Nemours and Company, Savannah River Laboratory, Aiken, SC, August 1991.

15 Cramer, D.S. Data Base Development and Equipment Reliability for Phase 1 of the Probabilistic Risk Analysis. DPST-87-642, E.I. du Pont de Nemours and Company, Savannah River Laboratory, Aiken, SC, October 1987.

16 OREDA, Offshore Reliability Data Handbonk. 1st Edition, OREDA, Hovik, Norway, 1984.

17 IEEE Guide to the Collection and Presentation of Electrical, Electronic, Sensing Component, and Mechanical Equipment Reliability Data for Nuclear-Power Generating Stations. IEEE, Std 500-1984, The Institute of Electrical and Electronics Engineers, Inc., New York, New York, December 1993.

18 Tinnes, S.P. Probabilistic Risk Assessment of SRS Reactor Scram Channel Wiring Failures (U). WSRC-RP-90-1308, Westinghouse Savannah River Company, Aiken, SC, November 1990.

19 Cramer, D.S. Eires Plus Explosions in Qil Filled Electrical Transformers (U). Calc-Note Q-CLC-G-00008, Rev. 0, Westinghouse Savannah River Company, Aiken, SC, November 1994.

20 IWP Methodology Manual for IWP Implementation. (Draft, to be issued October 1995)

21 Wellmaker, K.A. Determination of Frequencies of Tritium Releases and Fire Related Incidents for the Tritium Facilities SAR(U). WSRC-TR-950262, Westinghouse Savannah River Company, Aiken, SC, July 1995.

22 Jansen, J.M. and Mason, C.L. Erequency of Deflagration in the In-Tank Precipitation Process Tanks Due to Less of Nitrogen Purge System (U). WSRC-TR-93-169, Rev. 2, Westinghouse Savannah River Company, Aiken, SC, January 1994.

23 Lee, M.W., and Prout, W.E. Statistical Analysis of Sand Filter Etficiency. DPST-79-506, E.I du Pont de Nemours and Company, Savannah River Laboratory, Aiken, SC. September 1979.

24 Spangler, B.S. Chlorine Hazard to SRS Reactor Areas. WSRC-RP-1278, Westinghouse Savannah River Company, Aiken, SC, October 1989.

25 Brandyberry, M.D. Analysis of the Impact of SRS Helicopter Operations on Reactor Safety (U). WSRC-TR-90-90, Westinghouse Savannah River Company, Aiken, SC, March 1990.

26 Sarrack, A.G. Heliconter Crash for DWPE. Calc-Note S-CLC-S-00039, Westinghouse Savannah River Company, Aiken, SC, February 1995.

27 Benhardt, H.C., Eide, S.A., Held, J.E., Olsen, L.M., and Vail, R.E. Savannah River Site Human Error Data Base Develonment for Nonreactor Nuclear Facilities (U). WSRC-TR93-581, Westinghouse Savannah River Company, Aiken, SC, February 1994.

28 Crowley, D.A. to Smith, J.A: Meteorite. E.I. du Pont de Nemours and Company, Savannah River Laboratory, Aiken, SC, July 1986. 
29 . Brandyberry, M.D., Cramer, D.S., and Logan, V.E. Analysis of the Erequency of Loss of Control Rod or Euel Assembly Cooling Due to Plugging (LCRC/LOEA) (U). WSRC-RP91-956, Westinghouse Savannah River Company, Aiken, SC, October 1991.

30 Sharp, D.A. to Ahlfeld, C.E. Tomade Hazards to Production Reactors at Savannah River Plant. DPST-86-579, E.I: du Pont de Nemours and Company, Savannah River Laboratory, Aiken, SC, July 1986.

31 Lux, C.R. Distance Exceedance Probability for Train Wrecks at SRS (U). Calc-Note SCLC-S-00056, Westinghouse Savannah River Company, Aiken, SC, August 1995.

32 Crowley, D.A. to Sharp, D.A. Pipeline Explosions and Volcanos as Accident Initiators. E.I. du Pont de Nemours and Company, Savannah River Laboratory, Aiken, SC, September 1986. 


\section{DISTRMUTION}

W.J. Johnson, 730-B

F. Beranek, 730-B

M.J. Hitchler, 992-1W

D.A. Sharp, 992-1W

M.L. Cowen, 992-1W

O.M. Ebra-Lima, 707-F

R.F. Bradley, 992-2W

V.F. Lamb, 992-1W

J.M. Low, 707-F .

- T.G.Campbell, 707-F

D.F. Paddleford, 992-1W

J.A. Radder, 992-1W

J.H. Starling, 992-1W

R.E. Vail, 992-1W

C.R. Lux, 992-1W

D.S. Cramer, 992-1W

K.A. Wellmaker, 992-1W

S.R. Salaymeh, 992-1W

W.J. Copeland, 992-1W

D.W. Heal, 992-1W

G.L. Hovis, 992-1W

S.A.. Martin, 992-1W

I.K. Paik, 992-1W

S.M. Patel, 992-1W

D.E. Rossi, 992-IW

C.E. Shogren, 992-1W

C..H. Blanton, 992-1W

H.A. Ford, 992-1W

M.L. Moore, 773-A

E. Nomm, 992-1W

A.G. Sarrack, 992-1W

R.P. Taylor, Jr., 992-1W 